Instituto Internacional de Investigación y Desarrollo Tecnológico Educativo INDTEC, C.A.

DOI: https://doi.org/10.29394/scientific.issn.2542-2987.2016.1.2.11.181-199

OAI-PMH: http://www.indteca.com/ojs/index.php/Revista Scientific/oai

\title{
Formación del Docente de Educación Media General desde el Enfoque por Competencias
}

\author{
Autora: María Edixa Araujo Riveros \\ Universidad Nacional Experimental "Rafael María Baralt", UNERMB \\ mariaedixa9@hotmail.com \\ Zulia, Venezuela
}

\section{Resumen}

La presente investigación tiene como propósito analizar la formación de los docentes de educación media general desde el enfoque por competencias de los profesores pertenecientes al Núcleo Escolar Rural No 465 ubicado en el municipio Baralt del estado Zulia. Para ello se sustentó teóricamente en aportes de autores como Pérez (2010), Rychen (2012), Rueda (2011), entre otros. Metodológicamente fue descriptiva, analítica, con diseño de campo, no experimental, transeccional. La población estuvo conformada por 44 sujetos: 06 directivos, 38 docentes, pertenecientes a las instituciones seleccionadas. Se utilizó la encuesta para recolectar los datos mediante un cuestionario con escala tipo Likert, estructurado por cinco alternativas y 36 ítems. La validez se obtuvo por el juicio de siete expertos, la confiabilidad se calculó mediante el coeficiente Alfa de Cronbach obteniéndose un valor 0,8568 . Se concluyó que los docentes muestran una formación con predominio del modelo técnico, donde están ausentes las teorías pedagógicas actuales, sin dominio de las competencias necesarias para desarrollar su praxis. Se recomienda replantear sus funciones reconduciéndolas con base en la ejecución de competencias para construir un horizonte colectivo que le permita producir cambios sustantivos en su práctica diaria.

Palabras clave: formación docente; enfoque por competencias; educación media general.

Fecha de Recepción: 11-08-2016 Fecha de Aceptación: 05-09-2016 


\title{
Training of Teachers of Education General Media from the Competence Approach
}

\begin{abstract}
This research aims to analyze the training of teachers of general secondary education from the approach by competencies of teachers belonging to the Core Rural School No. 465 located in the municipality Baralt, Zulia state. For it was based on theoretical contributions of authors like Perez (2010), Rychen (2012), Wheel (2011), among others. Methodologically it was descriptive, analytical, with field design, not experimental, transeccional. The population consisted of 44 subjects: 06 directors, 38 teachers belonging to the selected institutions. The survey was used to collect data using a questionnaire with Likert scale, structured by five alternatives and 36 items. The validity was obtained by the trial of seven experts, the reliability was calculated by Cronbach Alpha obtained a value 0.8568. It was concluded that teachers show a predominance of technical training model, where current educational theories are absent, no domain needed to develop their skills with practice. It is recommended to rethink their reconduciéndolas functions based on the execution of skills to build a collective horizon that allows you to produce substantive changes in your daily practice.
\end{abstract}

Keywords: teacher training; competence approach; general secondary education.

Date Received: 11-08-2016

Date Acceptance: 05-09-2016 


\section{Introducción}

La educación, sin la menor duda, es uno de los factores esenciales para el desarrollo del potencial humano, tanto a nivel individual, como en su incidencia en el progreso colectivo de los pueblos, la base para el desarrollo social y cultural sostenible, el crecimiento económico, además de la democracia, factor decisivo para el desarrollo humano con incidencia sobre la vida política, social, cultural, económica y democrática.

En ese sentido, es el eje alrededor de la cual gira un concepto amplio del bienestar humano; por esa razón, se requiere de profesores con competencias para visualizar las complejidades propias de una realidad cambiante y demandante de capacidades que permitan entender mejor las necesidades actuales, visualizar tendencias, tomar decisiones en contextos de incertidumbre e identificar oportunidades, nuevos recursos para alcanzar la meta impuesta por la sociedad: la calidad educativa.

Por esa razón, quienes imparten enseñanza en la educación media general requieren el dominio de un conjunto de competencias, las cuales les permitan asumir los cambios propuestos en la educación venezolana, donde se incluyeron elementos novedosos que hasta el momento, sólo se implementaban en el nivel primario.

Las competencias se conciben como una metodología en la cual se pondera el saber hacer, visto no desde un enfoque conductista que se limita al desarrollo de acciones o tareas, que tengan una evidencia medible, cuantificable, observable, sino que tiene su fundamento en los principios constructivistas y del aprendizaje significativo; no es el hacer por hacer, o conocer simplemente como acumulación de saberes. Es un saber hacer en la práctica, pero motivado en un aprendizaje significativo que se transfiere a situaciones de la vida real y que implica la resolución de problemas en la práctica. 
De esta manera, el enfoque de competencias busca aportar una serie de repertorios de comportamientos, en los cuales el individuo pone en práctica, de forma integrada, aptitudes, rasgos de personalidad y conocimientos adquiridos, es decir, implica un saber hacer o actuar en forma responsable, válida en un contexto profesional particular, que, trasladado al desempeño académico de los profesores se refiere a la valoración de conocimientos, habilidades, capacidades expresadas en el saber, el hacer, así como el saber hacer.

Es decir, demostrar conocimiento, así como dominio del contenido a enseñar, saber cómo hacer llegar ese saber a través de la didáctica, constituyéndose, de esta manera, la competencia profesional, la cual viene definida, no tanto en función de los conocimientos teóricos, sino en la habilidad o capacidad inteligente de resolver problemas en situaciones difíciles, nuevas, únicas, propias de un entorno social complejo, cambiante, dinámico.

El desarrollo de estas competencias depende de una docencia centrada en el aprendizaje en la cual, a partir del uso de estrategias variadas, vinculadas con la realidad. En ese sentido, ser competente implica tener la atribución para tratar acerca de algo o resolverlo, ser experto o tener conocimiento sobre alguna situación.

Dentro de ese contexto se ubica la presente investigación dirigida a analizar la formación de los docentes de educación media general desde el enfoque por competencias de los profesores pertenecientes al Núcleo Escolar Rural N ${ }^{\circ} 465$ ubicado en el municipio Baralt del estado Zulia

Dentro del territorio zuliano se encuentra el municipio Baralt, en donde esa realidad se hace más evidente, sobre todo en los profesores de educación media general pertenecientes al Núcleo Escolar Rural № 465, quienes, en su mayoría fueron formados antes de la actual reforma educativa, cuya formación profesional quizás no se adecúe a los nuevos paradigmas educativos, los cuales requieren un cambio en los modelos formativos que contribuya a 
redimensionar el papel social de los docentes como agentes de transformación.

Visto de esa forma, se observa en la praxis de los profesores mencionados un desfase con los lineamientos emanados del ente rector, pues pareciera que su formación no estuvo orientada a la atención de los requerimientos de la sociedad venezolana, evidenciándose en visitas reiteradas de la investigadora, las siguientes debilidades: Ausencia de trabajo comunitario, falta de promoción del trabajo cooperativo, praxis cerrada ante los cambios propuestos por el Currículo Bolivariano, evidente desadaptación a las nuevas situaciones sociales, aparente desconocimiento de estrategias, habilidades y competencias que le permitan aprovechar los recursos del medio para aplicarlos a la construcción de conocimientos de la disciplina correspondiente.

Cabe señalar que, de continuar esa realidad, se estarán egresando bachilleres con escasa adaptación a la realidad social venezolana, donde se promueve la solidaridad y la cooperación, lo cual a su vez, propiciará ciudadanos (as) resistentes a los cambios propuestos, quienes ingresen a la educación universitaria en condiciones de desigualdad con sus compañeros. Por lo antes señalado, esta investigación pretende analizar la formación de los docentes de educación media general desde el enfoque por competencias de los profesores pertenecientes al Núcleo Escolar Rural № 465 ubicado en el municipio Baralt del estado Zulia.

\subsection{Formulación del problema}

Los planteamientos anteriores conducen a formular la siguiente interrogante:

¿Cómo debe ser la formación de los docentes de educación media general desde el enfoque por competencias de los profesores pertenecientes al Núcleo Escolar Rural № 465 ubicado en el municipio Baralt del estado Zulia? 


\section{Teoría y Conceptos}

\subsection{Objetivo general}

Analizar la formación de los docentes de educación media general desde el enfoque por competencias de los profesores pertenecientes al Núcleo Escolar Rural $N^{\circ} 465$ ubicado en el municipio Baralt del estado Zulia.

\subsection{Objetivos específicos}

Identificar los modelos de formación docente aplicados en la educación venezolana bajo los cuales fueron formados los profesores que laboran en los liceos adscritos al Núcleo Escolar Rural 465 ubicado en el municipio Baralt.

Describir las teorías pedagógicas que sustentan la formación docente de los profesores que laboran en los liceos adscritos al Núcleo Escolar Rural 465 ubicado en el municipio Baralt.

Presentar un constructo teórico sobre las competencias docentes que deben poseer los profesores que laboran en los liceos adscritos al Núcleo Escolar Rural 465 ubicado en el municipio Baralt.

Cabe resaltar, que para sustentar este artículo; se hizo necesario realizar una revisión bibliográfica y documental, de la cual surgió un conjunto de estudios referidos a las variables antes mencionadas, seleccionándose los siguientes:

La educación es un fenómeno complejo, esencialmente humano, en permanente cambio, determinado por un conjunto de variables en el cual intervienen diversos actores, y en donde, la formación de docentes es considerada un asunto focal, para el avance de políticas educativas coherentes con los planes de desarrollo del país. Para lograr lo anterior es indispensable que el docente posea el dominio de un conjunto de competencias tanto genéricas como específicas de su profesión, las cuales le permitan desempeñarse adecuadamente en su praxis diaria, de manera de 
cumplir con los requerimientos del nuevo modelo educativo propuesto en el Currículo Bolivariano.

Ahora bien, son diversos los estudios realizados sobre las variables: formación docente y enfoque por competencias, de los cuales se seleccionaron los siguientes: el estudio de Barrios (2013) denominado "Formación docente en el Sistema Educativo Bolivariano", de carácter documental, donde señalan que el avance del proceso revolucionario que vive el país a partir del año 1999, producto de los profundos cambios generados por la elaboración, presentación y posterior aprobación por la Asamblea Constituyente de un nuevo marco constitucional; el cual se orienta a la consolidación de una sociedad humanista, democrática, protagónica, participativa, multiétnica, pluricultural, entre otros, donde el pueblo tenga mayor participación en el proceso de construcción de un nuevo modelo de sociedad basada en el pensamiento de Simón Bolívar.

Se concluye que en Venezuela la formación de educadores revela la existencia de considerables desajustes y patrones alejados de la realidad vivida por la sociedad. Esto se observa en las insuficiencias presentadas por los profesionales de la educación el su desempeño en el sistema educativo bolivariano, las cuales se manifiestan a través de carencias en el desarraigo cultural, falta de pertinencia educativa del sistema, la especificidad de la crisis de legitimidad escolar, limitaciones del currículo existente, falta de compromiso de las instituciones educativas con la transformación de la realidad. La investigación anterior se seleccionó como antecedente por cuanto se refiere a la formación docente específicamente en Venezuela, señalando que la misma no sólo comprende los aspectos pedagógicos sino, adquirir dominio de otros elementos como la asunción de la cultura local, aportando elementos relacionados con ésta, así como la falta de compromiso de las instituciones educativas, considerados esenciales en la educación actual, los cuales podrían tomarse para fundamentar el estudio. 
Asimismo, se tomó en consideración la investigación de Oviedo (2013) titulada "Formación del docente en investigación educativa en las escuelas bolivarianas" la cual tuvo como objetivo proponer un plan de acción orientado a la formación en investigación educativa del personal docente de las escuelas bolivarianas del municipio Santa Rita, estado Zulia.

\subsection{Formación docente}

La formación docente se concibe como una acción permanente donde el profesor es responsable de la misma y la docencia es concebida como una tarea colectiva, contextualizada. Se trata de que el educador sea el motor generador de sus propios cambios e innovaciones educativas, mediante la reflexión crítica, reflexiva de sus actuaciones en colaboración con sus compañeros.

En ese sentido, la formación docente es una pieza clave en la renovación e innovación pedagógica orientada a la mejora de la calidad de la enseñanza, por lo tanto, la acción debe hacerse con los profesores y también haciendo investigaciones educativas con ellos. Por tanto, O'Connor y Seymour (2010:3) señalan:

La formación es el proceso que amplifica el aprendizaje y proporciona un contexto para el mismo en tres terrenos principales:

a. El conocimiento y cómo aplicarlo en la resolución de problemas

b. Aprendizaje de habilidades, involucra la experiencia práctica que es esencial para el desarrollo de habilidades que van desde las físicas hasta las interpersonales como la formación

c. Aprendizaje de valores y actitudes que se siente y se transmite en el convivir diario.

De acuerdo con lo antes expuesto, la formación debe conducir al docente a la adquisición del basamento teórico que apoye su compromiso de eventos al proceso complejo y dinámico de enseñanza-aprendizaje, el cual 
continuamente exige reflexión, toma de decisiones, es decir, es una actitud ante la vida, así como el mejoramiento de la profesionalidad. Al respecto, Nordenflycht (2011:2) plantea:

Durante largo tiempo, la estrategia más utilizada ha sido el curso de perfeccionamiento, el que bajo esta denominación genérica, ha ofrecido respuestas prefabricadas a demandas o problemas de supuesta ocurrencia general, sin considerar la realidad ni el medio en que los docentes ejercen su labor, sin tomar tampoco debida cuenta de su heterogeneidad, sus expectativas o intereses.

En opinión de la investigadora, la incidencia de estos cursos en la práctica docente es mínima por cuanto son dictados de manera contextualizada sin lograr modificar la misma, pues sus destinatarios difícilmente llegan a incorporar en su repertorio pedagógico nuevas estrategias o comportamientos, aun cuando hayan sido diseñados y desarrollados de manera adecuada.

Ahora bien, para Fernández (2012) en la formación de docentes operan sistemas complejos de configuraciones relativamente articuladas (pedagógicas, ideológicas, psicosociales), los cuales suelen ser comunes, compartidas, por cuanto constituyen el resultado colectivo de condiciones sociales de producción que, a su turno, producen efectos, también colectivos, pues condicionan las prácticas docentes.

En esa línea de pensamiento, el Ministerio del Poder Popular para la Educación (2006:4) en su portal oficial especifica los programas y políticas de formación expresando:

La formación docente establece la conformación de equipos a nivel zonal, distrital, municipal y local, articulados en redes intra e intersectoriales. Sus funciones son de carácter técnico pedagógico y sus integrantes pueden ser representantes de cada nivel y modalidad del Sistema Educativo y de las organizaciones educativas. La planificación de Formación Permanente se deriva de las necesidades detectadas, acorde 
con los intereses y el contexto social en la cual tenga su campo de acción. Construir la data de los recursos técnicos según los tópicos y las necesidades. Para ello, deberá realizar un diagnóstico, detectar las necesidades y potencialidades existentes dentro y fuera del plantel, distrito o municipio y estados, realizando el intervalo institucional; con estos datos, realizar un levantamiento de ubicación parroquial.

De la referencia anterior, se infiere que el Estado venezolano plantea una formación docente con una tendencia a la promoción del trabajo con la comunidad, entendiendo al profesor como propulsor de cambios sociales, concediendo importancia a valores tales como la cooperación y la solidaridad, siendo considerada como un asunto focal para el avance de políticas educativas coherentes con los planes de desarrollo del país.

\subsection{Modelos de formación docente}

En el campo educativo no es posible prescribir fórmulas que el docente esté llamado a seguir; pero se pueden proporcionar conceptos, generalizaciones para avivar la sensibilidad hacia ciertos eventos, problemas, posibilidades, los cuales pueden contemplarse y considerarse en la formación del mismo. Al respecto, Fernández (2012:24) refiriéndose al proceso de formación docente sostiene que es justamente en ésta:

... donde es posible distinguir visiones clásicas o románticas de la formación del profesorado, visiones modernas acordes con el rápido conocimiento acumulado desde los programas de investigación positivista, y algunas otras visiones contemporáneas de la formación donde están conviviendo diversos modelos paradigmáticos.

Al analizar la información anterior, se infiere que en un sistema educativo inclusivo como el venezolano, es indispensable garantizar una sólida formación profesional a los profesores de secundaria, basada en la diferentes disciplinas, como: psicología de la educación, pedagogía, sociología 
educativa, didáctica específica, las cuales les permitirán conocer cómo aprenden los estudiantes, los métodos de enseñanza necesarios para contextualizar el acto educativo, así como ser capaces de tomar decisiones sobre cómo transferir el conocimiento disciplinar a la dinámica del aula.

\subsection{Modelo académico}

Este modelo se corresponde, según Pérez (2010), con la orientación académica de la enseñanza, indicando la misma como un proceso donde se realiza la transmisión de conocimientos y se propicia la adquisición de la cultura pública; bajo la idea que la sociedad a lo largo de la historia es la encargada de desarrollar la información y los conocimientos necesarios, los cuales deben ser aprendidos por los alumnos, quienes fungen como meros recipientes, en donde el profesor vierte el bagaje de contenidos.

De acuerdo con lo anterior, lo esencial de un profesor es su sólido conocimiento de la (s) disciplina (s) impartidas a sus estudiantes. La formación, llamada pedagógica, pasa entonces a un segundo plano y suele considerarse superficial e innecesaria. Por lo tanto, sus saberes pueden conseguirse con la experiencia directa en los establecimientos, si se considera que cualquier persona con estudios, así como formación académica puede convertirse en educador.

\subsection{Modelo práctico}

Este modelo considera que la formación docente se basa prioritariamente en el aprendizaje de la práctica, para ella y desde éste, es decir, la experiencia obtiene fundamental trascendencia. Contempla la enseñanza como una actividad en donde no se pueden dictar recetas ni normas a través de las cuales se tenga la posibilidad de obtener los resultados deseados. 
Esta visión de la enseñanza, explica Colom (2011), como una actividad compleja, la cual se desarrolla en escenarios singulares, determinada por el contexto, regularmente con resultados imprescindibles y un cúmulo de juicios de valor, son los elementos que dan vida a este modelo. A partir de este modelo, se desarrolla el enfoque tradicional, el cual se trata de un tipo de formación profesional docente netamente conservador, en la que se concibe la enseñanza como una actividad totalmente artesanal, aprendida como resultado de un proceso de ensayo-error, en donde la relación entre el profesor y los estudiantes se considera el vehículo más apropiado para transmitir los conocimientos del educador de manera no reflexiva, intuitiva, como evidente producto de la rutina dentro del aula.

Lo anterior conlleva la mimetización de los profesores no novatos y la reproducción de vicios generados a lo largo del desarrollo de las instituciones de educación secundaria, así como el desenvolvimiento de los docentes veteranos. Visto de esa forma, esta visión considera el conocimiento profesional como una transmisión generacional, siendo el producto de un largo proceso de adaptación a los centros educativos, además, a la función de socialización.

\section{Metodología}

\subsection{Enfoque epistemológico}

El presente estudio tiene como enfoque epistémico metodológico para su realización los siguientes aspectos:

El Positivismo como posición filosófica, ya que la investigación a partir del movimiento de los hechos en situaciones concretas y objetivas permitirá recopilar informaciones científicamente. Esta apreciación concuerda con lo señalado por Lowy (2011:35) quien expresa "el positivismo es un método de investigación en la cual el científico debe desprenderse de prejuicios y 
presunciones, separar los juicios de hechos de los de valor, la ciencia de la ideología.

En ese sentido, la investigación pretende explicar la realidad basándose en la creencia que existe la esencia propia del objeto de conocimiento, pues el enfoque epistemológico positivista supone una realidad dada, la cual puede ser conocida de manera absoluta por el sujeto cognoscente.

\subsection{Tipo de investigación}

El tipo de investigación fue analítica, pues en el estudio de la problemática objeto de indagación, referida a la formación docente desde el enfoque por competencias de los profesores de los liceos adscritos al Núcleo Escolar Rural 465 ubicado en el municipio Baralt, se evidenciaron sistemáticamente las manifestaciones de las dimensiones e indicadores en forma independiente, para reconocer las magnitudes de aparición de aquellas en la formación del problema.

De igual forma, Hurtado (2010:56) expresa que el propósito de un estudio analítico "es entender las situaciones en términos de las relaciones de sus componentes, implica la reinterpretación de lo analizado en función de algunos criterios", tal como se pretende en la presente investigación, en la cual se analizara la formación de los docentes, con el fin de conocer, describir e interpretar la praxis que desarrollan en sus instituciones.

\subsection{Técnica e instrumento de recolección de información}

La técnica utilizada en el presente estudio fue la encuesta por considerar que la misma podía usarse para recabar datos vinculados con las opiniones de la población en torno a la frecuencia con que se presenta el fenómeno investigado relacionado formación docente desde el enfoque por competencias de los profesores de los liceos adscritos al Núcleo Escolar Rural 465 ubicado en el municipio Baralt. Al respecto, Finol y Camacho (2012:69) 
expresan "la encuesta es una herramienta utilizada por el sujeto investigador para recabar información acerca del hecho, evento o fenómeno que investiga".

Asimismo, Sabino (2010:71) señala que la encuesta trata de una técnica para "requerir información a un grupo socialmente significativo de personas acerca de los problemas en estudio para luego, mediante un análisis de tipo cuantitativo, sacar las conclusiones que se correspondan con los datos recogidos".

En cuanto al instrumento de recolección de información, este es definido por Rodríguez y Pineda (2011) como un recurso de que se vale el investigador para acercarse a la realidad y extraer información sobre su objeto de estudio. Con base en lo anterior, se elaboró para la presente investigación un cuestionario estructurado con cinco alternativas de respuesta para cada ítem: Siempre (5), Casi siempre (4), Algunas Veces (3), Casi Nunca (2), Nunca (1), los cuales sirvieron para obtener las respuestas emitidas por la población participante a las afirmaciones propuestas en el mismo.

\section{Resultados del Análisis de Contenido.}

El presente aspecto, hace referencia al análisis y discusión de los resultados obtenidos del proceso de recolección de la información a través del cuestionario aplicado al personal directivo y docente adscritos a los liceos pertenecientes al Núcleo Escolar Rural 465 ubicado en el municipio Baralt; los mismos son mostrados de acuerdo al orden de presentación de las variables formación docente y enfoque por competencias con sus respectivas dimensiones e indicadores.

\subsection{Análisis de los datos}

Para analizar los datos, el procedimiento aplicado fue totalizar los puntajes alcanzados por cada alternativa por indicador, posteriormente se dividió entre el total de la población de directivos y docentes multiplicado por 
el total de ítems que miden el indicador para extraer el porcentaje correspondiente al mismo, seguidamente esos porcentajes se promediaron para obtener los valores de cada dimensión. Asimismo, se procedió a extraer el promedio de las frecuencias relativas para determinar los correspondientes a cada variable. Estos pasos se realizaron con la finalidad de hacer más comprensible el análisis para el lector. Además, se expone la opinión de la investigadora basada en los referentes teóricos previamente analizados, los cuales permitieron la elaboración de las conclusiones y recomendaciones de la investigación, con el fin de lograr una información científica para Analizar la formación de los docentes de educación secundaria desde el enfoque por competencias de los profesores pertenecientes al Núcleo Escolar Rural № 465 ubicado en el municipio Baralt del estado Zulia.

\section{Conclusiones}

En atención al análisis de los datos y tomando como orientación los objetivos de la investigación, se derivan las siguientes conclusiones. Con respecto al primer objetivo específico identificar los modelos de formación docente aplicados en la educación venezolana bajo los cuales fueron formados los profesores que laboran en los liceos adscritos al Núcleo Escolar Rural 465 ubicado en el municipio Baralt se determinó que existen coincidencias en relación a las respuestas emitidas por ambas poblaciones cuando directivos y profesores señalan que éstos últimos siempre desarrollan un proceso transmisivo de enseñanza considerando que los estudiantes deben aprender mecánicamente los contenidos, por creer que solo es indispensable el aprendizaje de las asignaturas, el cual se toma como el logro de objetivos conductuales, reproduciendo así los modelos de enseñanza ya consagrados.

Asimismo, coinciden en señalar que los profesores casi nunca desarrollan una enseñanza basada en el aprendizaje de la práctica, escasamente promueven la construcción de conocimiento en sus estudiantes 
mediante la reflexión o adaptan la enseñanza a las necesidades de los estudiantes.

Se concluye que los profesores de las instituciones mencionadas recibieron una formación docente tradicional, como venía impartiéndose en las universidades venezolanas, donde lo fundamental era el dominio de las asignaturas que iban a impartir, mostrando predominio del modelo técnico, pues basan su enseñanza en el logro de los objetivos conductuales, mediante un proceso transmisivo en el cual los educandos deben aprender mecánicamente los contenidos.

En lo concerniente al objetivo específico dirigido a describir las teorías pedagógicas que sustentan la formación docente de los profesores que laboran en los liceos adscritos al Núcleo Escolar Rural 465 ubicado en el municipio Baralt se evidenció que ambas poblaciones coincidieron en que los profesores casi nunca toman en cuenta los postulados del cognitivismo, constructivismo, humanismo o teoría crítica, siendo la teoría humanista para la que mejor adaptan a su praxis, según explican los profesores, pues siempre capacitan a los estudiantes para el trabajo productor promoviendo en ellos la transformación social, sin embargo, para los directivos ésta nunca se evidencia en la praxis docente.

Se concluye que la formación recibida por los profesores, estuvo signada por un enfoque conductual clásico, alejado de las posturas teóricas plasmadas en el Diseño Curricular Bolivariano donde se plantea la enseñanza como un proceso de mediación en el cual el profesor se convierte en mediador entre los contenidos y el estudiante, facilitando estrategias que propicien la construcción de conocimientos mediante los saberes previos, así como la interacción con el entorno.

En lo concerniente al objetivo específico dirigido a caracterizar las competencias que poseen los profesores que laboran en los liceos adscritos al Núcleo Escolar Rural 465 ubicado en el municipio Baralt se demostró que 
existen discrepancias en las respuestas emitidas por ambas poblaciones, por cuanto los directivos señalaron que los profesores casi nunca muestran dominio de las competencias laborales, pues en raras ocasiones integran el saber y el hacer en su práctica cotidiana, afirmación evidenciada en el hecho que escasamente desarrollan sus clases de maneta teórico-prácticas.

Asimismo, manifestaron que los profesores casi nunca promueven el trabajo en equipo en sus estudiantes, mostrando escasa sensibilidad para adaptarse a las necesidades de éstos; además, consideran que éstos nunca dominan las competencias propias de la función docente, pues no comunican claramente a sus estudiantes los objetivos que deben lograr, tampoco utilizan la investigación como estrategia de enseñanza ni contextualizan el programa de la disciplina adaptándolo a sus necesidades.

Por el contrario, los docentes indicaron que algunas veces se adaptan a las necesidades de sus estudiantes, desarrollando habilidades en el desarrollo de estrategias novedosas en clase, además, en ocasiones adaptan su práctica a las diferentes situaciones que se presentan en el aula, aun cuando reconocen poseer debilidades en relación a las competencias docentes, pues raramente utilizan la investigación como estrategia de enseñanza, en escasas oportunidades contextualizan el programa de su asignatura para adaptarlo a las necesidades de sus educandos.

Se concluye que los profesores presentan debilidades en relación a su desempeño basado en competencias, desarrollando su praxis, tal como fueron formados, es decir, sin implementar las modificaciones exigidas en el Currículo Bolivariano donde se plantea un proceso de enseñanza basado en la mediación de los contenidos, a través de la contextualización del programa para adaptarlo a las necesidades del estudiante y la comunidad. 


\section{Referencias}

Barrios, L. (2013). "Formación docente en el Sistema Educativo Bolivariano". Trabajo de Ascenso. Universidad de Oriente. Barcelona.

Colom, A. (2011). "Las teorías de la posmodernidad y la educación". Editorial Ariel. Barcelona. España.

Fernández, M. (2012). “Desarrollo profesional docente”. Ediciones Grupo Universitario. Granada, España.

Finol, M. y Camacho, H. (2012). "El Proceso de Investigación Científica”. Editorial Ediluz. Maracaibo.

Hurtado, J. (2010). "El proyecto de investigación". Editorial Sypal. Caracas. Lowy, P. (2011). "Paradigmas Educativos". Editorial Anaya. Madrid.

Nordenflycht (2011). “Formación del profesorado". Editorial Graó. Barcelona, España.

O'Connor, S. y Seymour, P. (2010). "Formación de formadores". Editorial Anaya. Madrid.

Oviedo, D. (2013). “Formación del docente en investigación educativa en las escuelas bolivarianas". Trabajo de Ascenso. Universidad Valle del Momboy. Valera.

Rodríguez, H. y Pineda, A. (2011). "La aventura de investigar". Editorial Ecoe. Bogotá.

Rueda, S. (2011). "La evaluación del desempeño docente: consideraciones desde el enfoque por competencias". Revista Electrónica Actualidades Investigativas en Educación. Vol. 09, núm. 1, págs. 34-49. Universidad de Sonora. México.

Rychen, F. (2012). "Competencias profesionales docentes". Editorial Horsori. Madrid.

Sabino, C. (2010). "El proceso de la investigación". Editorial Panapo. Caracas. 


\section{María Edixa Araujo Riveros \\ e-mail: mariaedixa9@hotmail.com}

Nació en Pueblo Nuevo, Municipio Baralt, Estado Zulia, el 09/04/1976. Estudiante de la Universidad Nacional Experimental "RAFAEL MARÍA BARALT" en el DOCTORADO EN EDUCACIÓN. Estudios de postgrado: Universidad Nacional Experimental "RAFAEL MARÍA BARALT", título obtenido MSc. En Administración de la Educación Básica. Estudio de Pregrado: U.N.E.R.M.B; título Obtenido: LICENCIADA EN EDUCACIÓN MENCIÓN EDUCACIÓN INTEGRAL. Ha participado en cursos, talleres y simposios sobre Nuevo Enfoque de Educación Básica. UNERMB. Mapas Mentales y Conceptuales. Instrumentos de Evaluación Cualitativa. Para la I y II de Educación Básica. III Jornadas de Docentes Investigadores en Acción. Normas de Presentación de Trabajo Científico. U.N.E.R.M.B. La Comunicación Como Estrategia para la Construcción de Equipos de Trabajos Productivos. Gerencia Educativa. FUNDAIUTEMBI. EI Docente como Mediador - Nuevo Paradigma de la Educación. Desarrollo del Pensamiento Lógico Matemático. Paradigmas de la Investigación de la Educación. El Proyecto Educativo Integral Comunitario (PEIC) Como Herramienta en la Integración Escuela- Comunidad. La Escuela para Familia como Estrategia para Mejorar la Calidad Educativa en la E.E.C. "Loma del Medio". El Rol del Gerente para la Integración de los Padres y Representantes. Experiencia laboral: Escuela Básica Municipal "El Boquete", Sector El Boquete - Baralt, Zulia docente de aula (2 años). Docente universitario en la UBV Misión Sucre en la aldea Dr. Jesús María Portillo Anexo El Batatal (6 años). U.E.N "El Batatal", NER 465 docente de CRA desde febrero 2015 hasta la fecha.

El contenido de este manuscrito se difunde bajo una Licencia de Creative Commons ReconocimientoNoComercial-Compartirlgual 4.0 Internacional 